\title{
Diseño y construcción de un convertidor dc/dc tipo Boost con PWM ajustable
}

\author{
Design and construction of a Boost type dc / dc converter with adjustable \\ PWM
}

\author{
Jesser James Marulanda Durango, Jhon Jairo Ordoñez, Luis Fernando Mosquera Machado \\ Facultad de ingenierías, ingeniería eléctrica, Universidad tecnológica de Pereira, Pereira, Colombia \\ jjmarulanda@utp.edu.co \\ jhonjoulhotmail.com \\ lfmosqueradutp.edu.co
}

\begin{abstract}
Resumen- En este artículo se presenta el diseño y los parámetros de construcción de un convertidor DC-DC tipo Boost con PWM ajustable, este proyecto se dividió en 4 etapas, en la primera etapa se realiza un estudio de los módulos experimentales de algunos convertidores de corriente, en la segunda se realizó el diseño del convertidor, en la tercera etapa se construye el módulo experimental (construcción del PWM ajustable, cuya finalidad es realizar cambios en tiempo real en el circuito, con frecuencia y ciclo de trabajo variable, construcción del circuito de potencia con las especificaciones dadas por último se diseña la carcasa del convertidor) en la cuarta etapa se analizan los valores teóricos y prácticos del módulo para su validación.
\end{abstract}

Palabras clave - Convertidor, conmutación, carga, frecuencia, PWM,

Abstract- For the design of the experimental prototype of the boost converter with adjustable PWM, this project was divided into 4 stages, the first stage of a study modules oriented teaching power converters in the second design of the converter is performed is performed in the third stage the experimental module (construction adjustable PWM is constructed, whose purpose is to make changes in real time on the circuit, frequency and variable duty cycle, construction of the power circuit with the specifications given finally designs converter housing) in the fourth stage the theoretical and practical module values are analyzed.

Key Word -Converter, Commutation, Load, Frequency, PWM

\section{INTRODUCCIÓN}

La electrónica de potencia influye en muchas aplicaciones, porque se encarga de la conversión de energía usando dispositivos electrónicos y técnicas de control que permiten un manejo eficiente de la energía. De manera sencilla un sistema de potencia, de acuerdo a su entrada o salida de voltaje podemos reconocer varias topologías: DC-DC, CD-CA (inversores), CA-CD (rectificadores), y CA-CA, los convertidores DC-DC se encargar de convertir un voltaje de suministro constante, a los niveles de voltaje o corriente que requiera la carga. En general los convertidores DC-DC poseen numerosas configuraciones, entre estas esta: convertidor elevador (Boost), convertidor reductor (Buck), y convertidor reductor-elevador (Buck-Boost). En estos circuitos un factor muy importante es el de la frecuencia de conmutación ya que de esta depende la eficiencia de estos convertidores [1]. Hoy en día los avances en la electrónica de potencia nos permiten diseñar y construir convertidores con técnicas de control moderno, número reducido de componentes, baja inversión, alta confiabilidad y muy buena eficiencia [2].

Por otro lado, en el laboratorio de ingeniería eléctrica hay una serie de dispositivos y herramientas que logran hacer que la práctica y la teoría vayan de la mano, siempre hay que unir esfuerzos entre la universidad y los estudiantes para así lograr un equilibrio entre la práctica y la teoría para así afianzar más el proceso de aprendizaje [3]. Para dar solución a algunas falencias se propone construir un módulo para un curso de electrónica de potencia que contenga un convertidor DC-DC Boost (elevador). Este módulo es una herramienta para reforzar los conceptos aprendidos en clase a través de la práctica, verificando el tiempo real el funcionamiento del circuito. 


\section{DISEÑO DEL CONVERTIDOR}

Para el diseño del convertidor DC-DC tipo Boost (elevador) se hace un análisis del modelo dinámico de este, basado en las leyes de Kirchhoff, aplicadas a todos los estados del circuito, en la figura 1 se muestra el modelo circuital del convertidor [4].

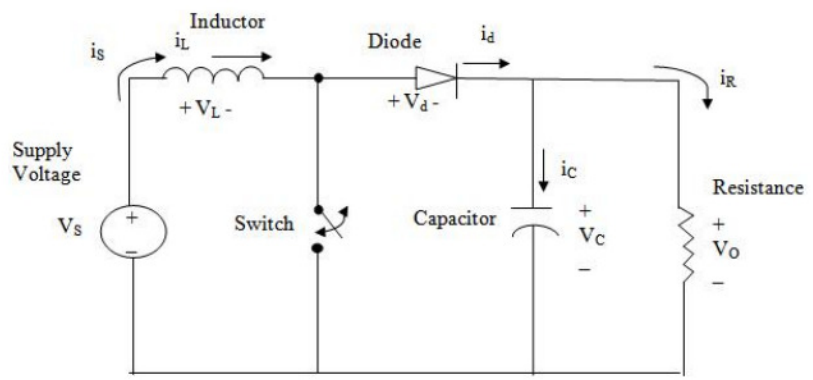

Figura 1. Topología del convertidor Boost

Básicamente el funcionamiento del convertidor se resume así:

- Cuando el interruptor (switch) está cerrado en un tiempo ton la bobina (L) almacena energía de la fuente, a la vez la carga (R) es alimentada por el condensador (C).

- Cuando el interruptor (switch) está abierto en un tiempo tof, el único camino para la corriente es a través del diodo, y circula por el condensador $\mathrm{C}$ y por último la carga.

Parámetros de diseño del convertidor DC-DC Boost.

$$
\begin{aligned}
& V o=\frac{V s}{1-D} \\
& \operatorname{Lmin}=\frac{D(1-D)^{\prime \prime} 2 R}{2 f} \\
& L=\text { FACTOR *Lmin } \\
& \operatorname{Imax}=\left(\frac{V s}{(1-D)^{\prime \prime 2} 2 R}+\frac{(V s D)}{2 L f}\right) \\
& \operatorname{Imax}=\left(\frac{V s}{(1-D)^{\prime \prime 2} 2}-\frac{(V s D)}{2 L f}\right) \\
& \mathrm{C}=\frac{(D)}{R T f \frac{\Delta V o}{V o}}
\end{aligned}
$$

Formas de onda del convertidor Boost.
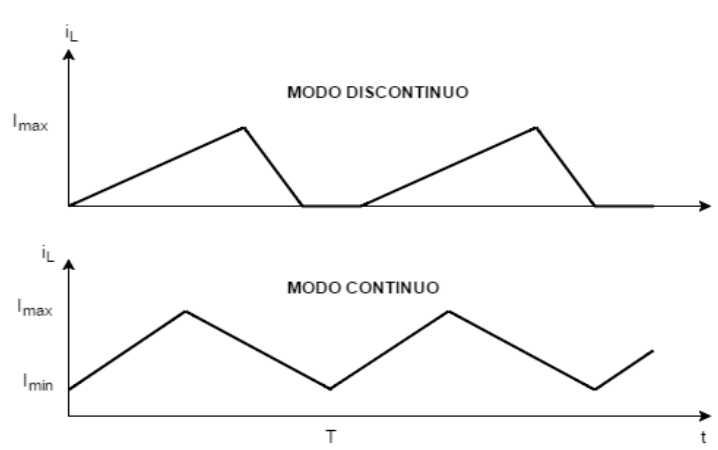

Figura 2. Formas de onda del convertidor Boost (CCM Y CDM)

\section{ESPECIFICACIONES DE DISEÑO}

\begin{tabular}{|c|c|}
\hline Parámetro & Valor \\
\hline Tensión de entrada & $9 \mathrm{~V}$ \\
\hline $\begin{array}{c}\text { Corriente Max de } \\
\text { salida }\end{array}$ & $2 \mathrm{~A}$ \\
\hline Inductancias & {$[50-500] \mathrm{uH}$} \\
\hline Resistencia & {$[50-100] \Omega$} \\
\hline $\begin{array}{c}\text { Frecuencia de } \\
\text { conmutación }\end{array}$ & {$[20-150] \mathrm{kHz}$} \\
\hline $\begin{array}{c}\text { Tensión Max. de } \\
\text { salida }\end{array}$ & $30 \mathrm{~V}$ \\
\hline
\end{tabular}

TABLA 1. Especificaciones de diseño

\section{CONSIDERACIONES DE DISEÑO}

- Si la frecuencia de conmutación aumenta, el tamaño del inductor y el condensador mínimo para la corriente continua limita el rizado de la tensión de salida.

- Altas frecuencias de conmutación permiten reducir la distorsión armónica total.

- L está diseñado para ser un $125 \%$ mayor que el valor mínimo calculado para la inductancia.

- El condensador se selecciona para limitar el rizado de de salida en $1 \%$ de las especificaciones de diseño para soportar la tensión máxima de salida.

- El interruptor seleccionado (Mosfet) debe soportar la tensión máxima cuando está apagado y la corriente mínima cuando está activo.

- El índice máximo de temperatura de cada uno de los componentes no debe ser superado.

\section{MODULACIÓN POR ANCHO DE PULSO O PWM}

Como ven en la figura 1 el transistor mosfet debe conmutar, el dispositivo PWM debe controlar la cantidad de veces que este cambie de estados [4]. La modulación por ancho de pulsos (también conocida como PWM, siglas en inglés de pulse-width modulation) de una señal o fuente de energía es 
una técnica poderosa en la que se modifica el ciclo de trabajo de una señal periódica (senoidal, triangular), para controlar circuitos analógicos con salidas digitales de un microprocesador [5].

El PWM se emplea en una amplia variedad de aplicaciones, que van desde equipos de medición hasta comunicaciones para el control de energía o potencia que se envía a una carga y la conversión [6]. En la figura 2 se muestran señales PWM con diferentes ciclos de trabajos para una mirada más afondo.

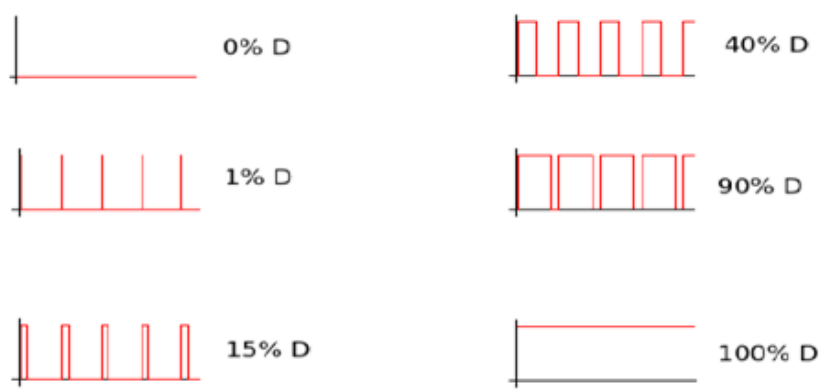

Figura 3. Topología del convertidor DC-DC tipo Boost.

La modulación por ancho de pulso se basa en la comparación de una señal de referencia a modular y una señal portadora de forma triangular o diente de sierra (figura 2). La comparación generará un tren de pulsos de ancho específico que se utilizan en la conmutación [6]. En la figura 3 se observa el proceso.

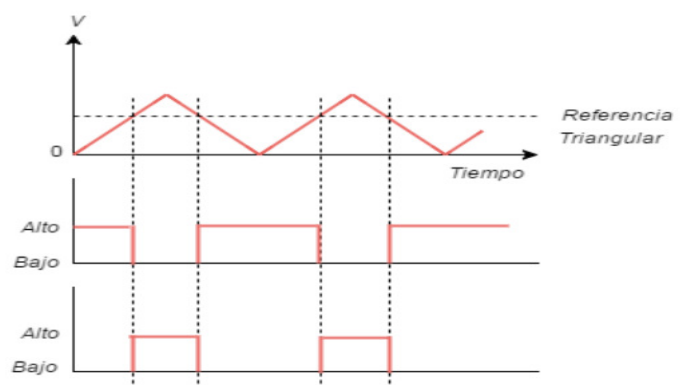

Figura 4. Topología del convertidor DC-DC tipo Boost

\section{CONSTRUCCIÓN DEL MÓDULO}

Para la construcción del módulo experimental del convertidor DC-DC Boost con PWM ajustable se procede a seguir una serie de paso como son:

\section{A. DESARROLLO DEL PWM AJUSTABLE.}

Para el desarrollo del PWM ajustable se utiliza el microcontrolador PIC16f877A, su función consiste en proporcionar un pulso de onda cuadrada con ciclo de trabajo y frecuencia variable, este pulso se le aplica a la puerta del transistor (mosfet).sus características tales como, memoria de programa, memoria de datos, frecuencia máxima de oscilación de $20 \mathrm{MHz}$, lo hacen versátil para el desarrollo del PWM ajustable. Debido a las características que nos brinda el microcontrolador PIC, podemos programar, lo que quiere decir, que se puede planificar la manera cómo va a funcionar nuestro PWM ajustable (adaptarse a nuestras necesidades). En la figura 5 se muestra un diagrama de bloques de como programar el microcontrolador PIC 16f877A y su respectiva salida PWM.

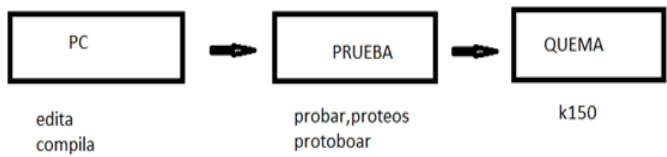

Figura 5. Diagrama bloques para programar el PIC 16F877A.

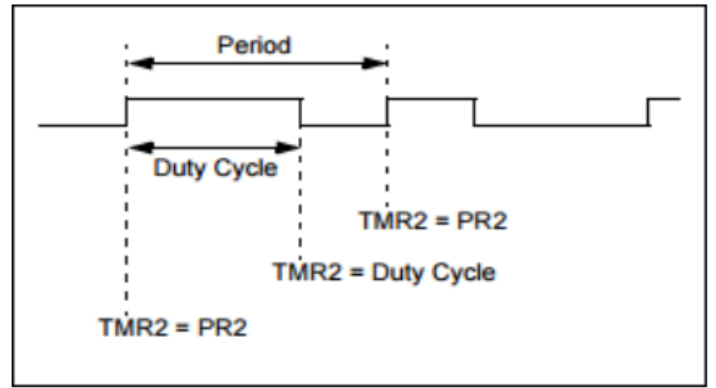

Figura 6. Salida PWM del PIC.

Después de haber realizado y compilado el código del PWM ajustable procedemos a montar el circuito en una protoboar con el fin de probar su funcionamiento. En la figura 6 y 7 se ilustra el esquema del circuito con PWM ajustable.

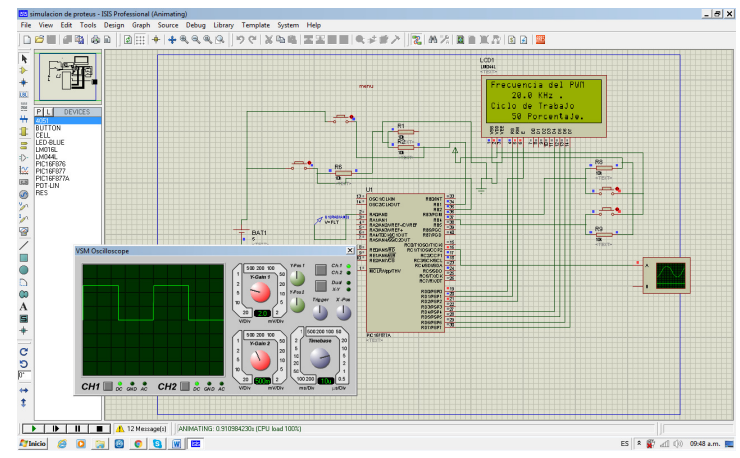

Figura 7.Prototipo del PWM ajustable montado en Proteus.

\section{B. CIRCUITO DE POTENCIA.}

Como ya sabemos la parte de potencia del convertidor realiza la conversión básica de energía del voltaje de entrada al voltaje de salida, incluyendo switches y el filtro de salida. Comúnmente en el circuito de potencia del convertidor se puede notar un transistor MOSFET de canal $\mathrm{N}$, un diodo denotado con la letra (D), capacitor (C) y un inductor (L), finalmente una resistencia $(\mathrm{R})$ que representa la carga. 
Durante el funcionamiento normal del convertidor, el MOSFET es encendido y apagado por el circuito PWM, este comportamiento switching crea pulsos en el circuito; el inductor está conectado al capacitor únicamente cuando el diodo conduce (se forma un filtro L/C), el mismo filtra el tren de pulso para producir un voltaje de salida DC. A continuación se muestran los elementos del circuito de potencia en la Tabla 2.

\begin{tabular}{|l|c|}
\hline \multicolumn{1}{|c|}{ Parámetro } & Valores \\
\hline Inductores & $50 \mathrm{uH}, 500 \mathrm{uH}, 800 \mathrm{uH}$ \\
\hline Capacitor & $330 \mathrm{uF}$ \\
\hline Diodo (diodo rápido) & FMLG 16S \\
\hline Mosfet & IRFZ44N \\
\hline Resistencia & $50 \Omega, 100 \Omega, 320 \Omega$ \\
\hline
\end{tabular}

TABLA 2. Elementos de potencia del convertidor Boost.

\section{DISEÑO DE LA CARCASA.}

Para la construcción del módulo experimental del convertidor DC-DC Boost con PWM ajustable, se utilizó una caja rectangular de plástico con el fin de aislar los conductores y demás elementos electrónicos, en las figuras 8,9 y 10 se ilustra lo anterior.

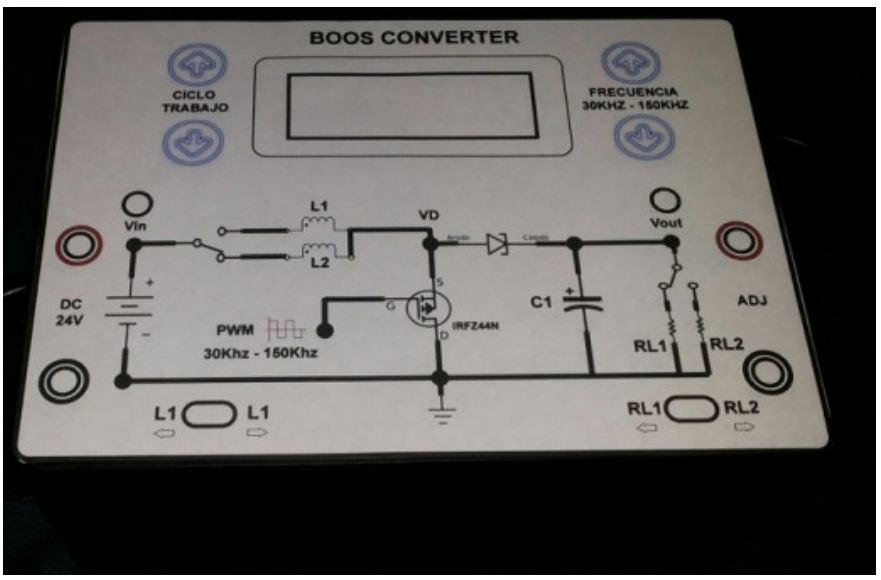

Figura 8. Diseño del adhesivo del módulo.

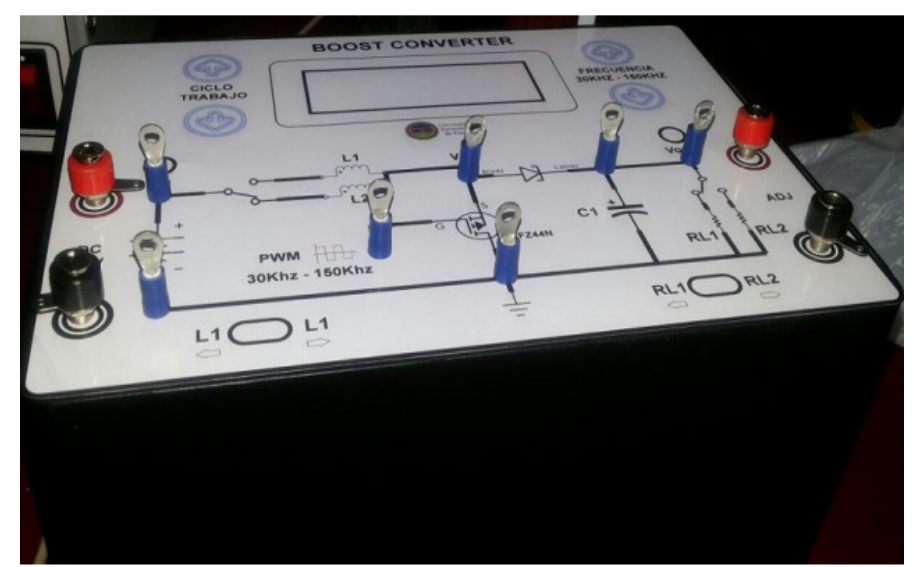

Figura 9. Distribución de pines del módulo.

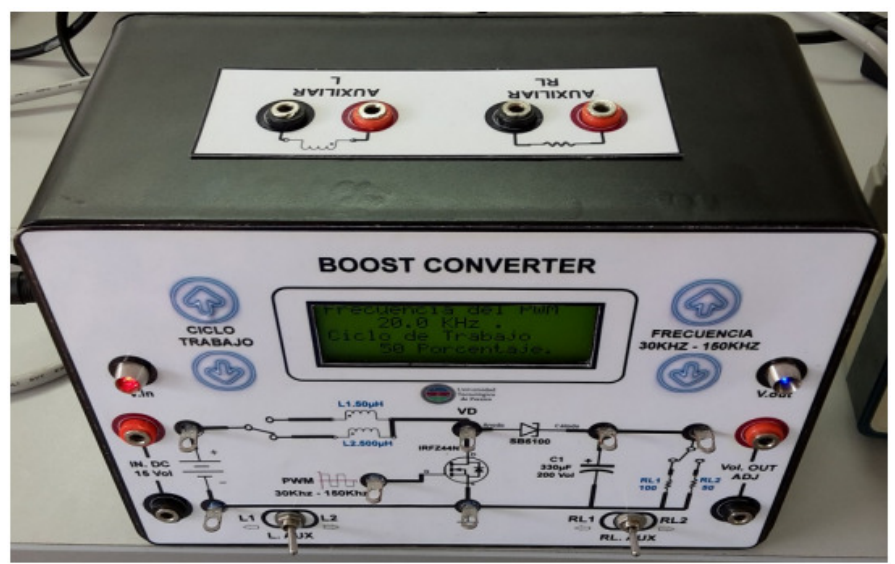

Figura 10. Módulo experimental ensamblado.

En la figura 10 se observa el módulo experimental del convertidor DC-DC Boost con PWM ajustable totalmente acoplado (acoplamiento del circuito de potencia y el circuito del PWM ajustable).

\section{ANALISIS DE RESULTADOS}

Luego de terminar la construcción del convertidor se realiza una simulación en MATLAB para tener una apreciación de su comportamiento. Esta simulación se hizo con todos los componentes del convertidor Boost. Se realizaron las pruebas necesarias para comprobar el correcto funcionamiento del circuito de potencia y del PWM, las pruebas están enfocadas en buscar dos puntos de operación del convertidor (operación en modo continuo y operación en modo discontinuo.

\section{Modos de operación continúa}

Estas pruebas se realizan con el fin de validar lo teórico con lo práctico y corroborar el funcionamiento del módulo con los puntos de operación que se muestran en la Tabla 3 se observan las especificaciones de diseño.

\begin{tabular}{|l|c|}
\hline \multicolumn{1}{|c|}{ Parámetro } & Valor \\
\hline Tensión de entrada & $9 \mathrm{~V}$ \\
\hline Corriente máxima de salida & $2 \mathrm{~A}$ \\
\hline Inductancias & {$[50-500] \mu \mathrm{H}$} \\
\hline Resistencia & {$[50-100] \Omega$} \\
\hline Frecuencia de conmutación & {$[20-150] \mathrm{KHz}$} \\
\hline
\end{tabular}

TABLA 3. Especificaciones de diseño del prototipo.

A continuación, se presentan una serie de tablas que muestran información del comportamiento del convertidor en modo continuo, donde se hace una comparación de los valores teóricos y prácticos del convertidor Boost, también se calcula 
su diferencia relativa para tener una mejor percepción para su respectivo análisis.

Diferencia relativa $=\frac{x-y}{f(X, Y)} * 100$

Donde $\mathrm{f}$ se ha escogido como el mayor número entre $\mathrm{x}, \mathrm{y}$.

En las Tablas 4, 5, 6 y 7 Con $\mathrm{R}=100 \Omega, \mathrm{L}=500 \mu \mathrm{H}$.

\begin{tabular}{|l|l|l|l|l|}
\hline $\mathrm{D}(\%)$ & $\mathrm{F}(\mathrm{kHz})$ & Vo(práctico) & Vo(teórico) & $\mathrm{D} . \mathrm{R}(\%)$ \\
\hline 25 & 30 & $10.82 \mathrm{~V}$ & $11.21 \mathrm{~V}$ & 3.4 \\
\hline 50 & 40 & $14.02 \mathrm{~V}$ & $17.14 \mathrm{~V}$ & 18.2 \\
\hline 60 & 50 & $15.08 \mathrm{~V}$ & $21.6 \mathrm{~V}$ & 30.18 \\
\hline
\end{tabular}

TABLA 4. Voltaje práctico vs voltajes teóricos

\begin{tabular}{|l|l|l|l|l|}
\hline $\mathrm{D}(\%)$ & $\mathrm{F}(\mathrm{kHz})$ & $\operatorname{Imax}($ práctico) & $\operatorname{Imax}($ teórico) & $\mathrm{D} . \mathrm{R}(\%)$ \\
\hline 25 & 30 & $0.316 \mathrm{~A}$ & $0.26 \mathrm{~A}$ & 17.7 \\
\hline 50 & 40 & $0.320 \mathrm{~A}$ & $0.57 \mathrm{~A}$ & 43.85 \\
\hline 60 & 50 & $0.368 \mathrm{~A}$ & $0.82 \mathrm{~A}$ & 55.12 \\
\hline
\end{tabular}

TABLA 5. Corriente máxima práctica vs corriente máxima teórico

\begin{tabular}{|l|l|l|l|l|}
\hline $\mathrm{D}(\%)$ & $\mathrm{F}(\mathrm{kHz})$ & $\operatorname{Imin}($ práctico) & Imin(teórico) & $\mathrm{D} . \mathrm{R}(\%)$ \\
\hline 25 & 30 & $0.264 \mathrm{~A}$ & $0.10 \mathrm{~A}$ & 62.12 \\
\hline 50 & 40 & $0.268 \mathrm{~A}$ & $0.33 \mathrm{~A}$ & 18.72 \\
\hline 60 & 50 & $0.272 \mathrm{~A}$ & $0.61 \mathrm{~A}$ & 55.40 \\
\hline
\end{tabular}

TABLA 6 Corriente mínima practica vs corriente mínima teórico

Los voltajes prácticos fueron tomados con el osciloscopio (ref. 2542B) y se corroboró con el flucke. En la tabla 7 se muestran otros valores en modo de operación continúa.

\begin{tabular}{|l|l|l|l|}
\hline $\mathrm{D}(\%)$ & Vo(teórico) & Vo(practico) & D.R (\%) \\
\hline 10 & $9.00 \mathrm{~V}$ & $9.18 \mathrm{~V}$ & 1.96 \\
\hline 20 & $9.20 \mathrm{~V}$ & $9.49 \mathrm{~V}$ & 3.05 \\
\hline 30 & $10.5 \mathrm{~V}$ & $11.1 \mathrm{~V}$ & 5.40 \\
\hline 40 & $12.1 \mathrm{~V}$ & $13.4 \mathrm{~V}$ & 9.70 \\
\hline 50 & $14.2 \mathrm{~V}$ & $16.2 \mathrm{~V}$ & 12.3 \\
\hline 60 & $17.3 \mathrm{~V}$ & $19.1 \mathrm{~V}$ & 9.42 \\
\hline 70 & $21.8 \mathrm{~V}$ & $21.9 \mathrm{~V}$ & 0.45 \\
\hline 80 & $28.9 \mathrm{~V}$ & $24.3 \mathrm{~V}$ & 15.9 \\
\hline 83 & $44.4 \mathrm{~V}$ & $27.7 \mathrm{~V}$ & 37.6 \\
\hline 85 & $53.1 \mathrm{~V}$ & $27.9 \mathrm{~V}$ & 47.5 \\
\hline 87 & $58.4 \mathrm{~V}$ & $27.6 \mathrm{~V}$ & 52.7 \\
\hline 90 & $68.4 \mathrm{~V}$ & $27.2 \mathrm{~V}$ & 60.0 \\
\hline 95 & $91.3 \mathrm{~V}$ & $25.7 \mathrm{~V}$ & 71.8 \\
\hline 100 & $\infty$ & $9.17 \mathrm{~V}$ & \\
\hline
\end{tabular}

Tabla 7. Voltajes teóricos vs voltajes prácticos a una frecuencia de 20 kHz.

En la tabla 7 la diferencia entre voltajes teóricos y práctico se debe a: Para los datos teóricos los componentes que se trabajan en la simulación son componentes ideales (no hay perdidas), y cada vez más que el ciclo de trabajo se acerca a la unidad o al $100 \%$ la tensión de salida se hará infinita; por otro lado los datos prácticos tomados del módulo son producidos por componentes reales (hay pérdidas asociadas debidos a los elementos parásitos de los elementos), debido a esto la tensión de salida nunca se hará infinita.

\section{ANALISIS DE INDUCTORES}

El convertidor Boost cuenta con dos inductores $(50 \mathrm{uH}-$ $500 \mathrm{uH}$ ), estos dos inductores nos permiten observar en qué modo de operación trabaja el convertidor, mediante las formas de onda de la corriente en el inductor (IL). En las figuras $11 \mathrm{y}$ 12 se observa el modo de operación del módulo construido.

En la figura 11 se ve la gráfica de la corriente en el inductor de $500 \mathrm{uH}$ del convertidor

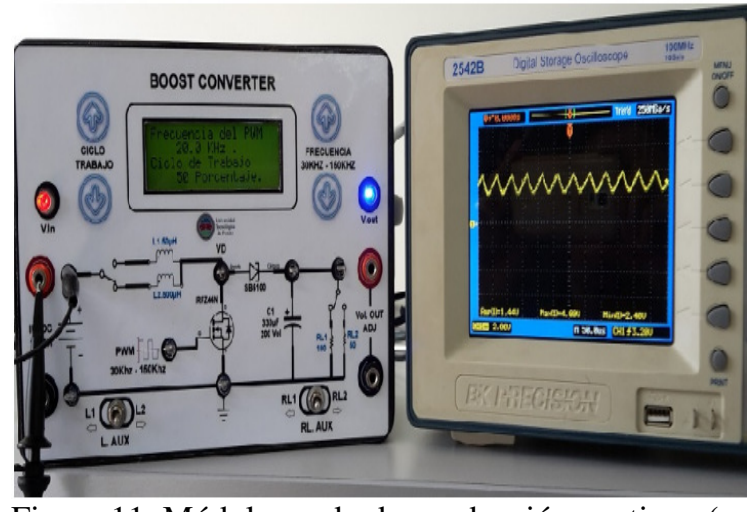

Figura 11. Módulo modo de conducción continua (practico)

En la figura 12 se muestra la gráfica del inductor dos de $50 \mathrm{uH}$

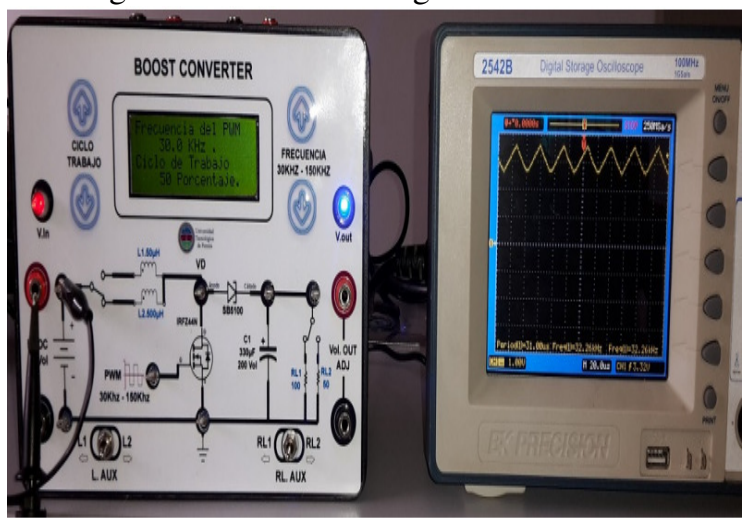

Figura 12. Módulo modo de conducción continua (práctico)

En la gráfica del inductor 11 y 12 se puede observar que el módulo está trabajando adecuadamente, ya que se encuentra trabajando en modo continuo por ende la corriente en el inductor nuca caerán a cero.

\section{MODO DE OPERACIÓN DISCONTINUA}

Con el fin de encontrar otros puntos de operación del convertidor se realizan otras pruebas. En ella se utilizan las salidas auxiliares con el fin de encontrar puntos de operación discontinua. En la figura se observa las gráficas del inductor 
(auxiliar) con un valor de $800 \mu \mathrm{H}$ y la carga de $320 \Omega$, donde se aprecia que el módulo está trabajando en modo discontinua.

Esta prueba se realiza utilizando las salidas auxiliares. Con $\mathrm{R}=100 \Omega, \mathrm{L}=800 \mu \mathrm{H}, \mathrm{D}=30 \%$ y f $=20 \mathrm{kHz}$.

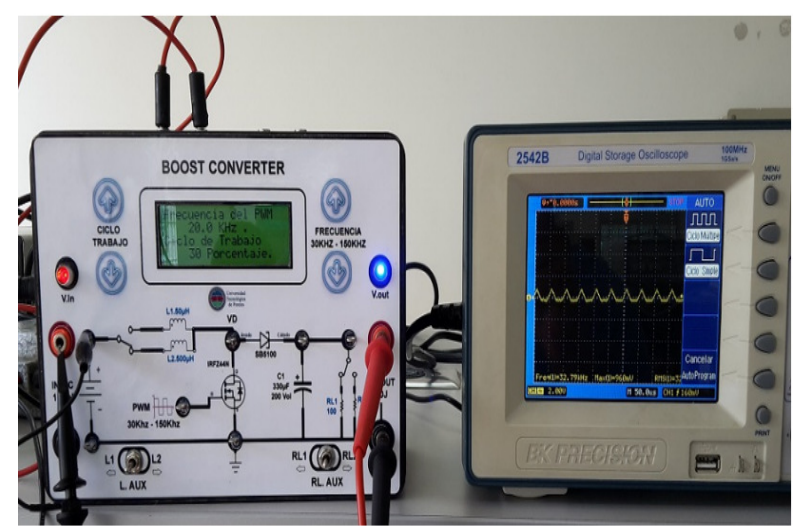

Figura 13. Módulo modo de operación discontinuo (práctico).

En la figura 13 se puede ver las gráfica del inductor 3, donde se observa que el sistema entra en modo operación discontinuo, la corriente que pasa por el inductor cae en un intervalo finito de tiempo. Otros puntos de operación en modo discontinuo se encuentran utilizando los puertos auxiliares del módulo con $\mathrm{R}=320 \Omega, \mathrm{L}=800 \mu \mathrm{H}$ y $\mathrm{R}=300 \Omega, \mathrm{L}=800 \mu \mathrm{H}$.

\section{CONCLUSIONES}

- Se ha cumplido el objetivo principal de este proyecto el cual fue la construcción del módulo convertidor CD-CD Boost con PWM ajustable, operando y funcionando adecuadamente, el cual será destinado al laboratorio de electrónica de potencia.

- Realizando un estudio teórico de IGBT"S Y MOSFET se permite tener criterios de selección para construir el circuito de potencia con el conmutador más adecuado para este convertidor.

- El convertidor propuesto se acopla completamente a un laboratorio de electrónica de potencia, tanto en sus entradas como en su funcionamiento, con los equipos existentes sin ningún requerimiento adicional.

\section{RECOMENDACIONES}

- Se recomienda utilizar como fuente DC, cargadores de computadoras o fuente de computadoras, debido a su corriente que estos cargadores admiten

- Se recomienda que el módulo experimental del convertidor Boost no esté conectado más de 4 horas en forma continua debido a los calentamientos de los dispositivos.

\section{REFERENCIAS}

[1]. Ortega, E. o. (2012). Desarrollo de convertidores DC-DC para supercapacitor con aplicacion en traccion electrica. Mexico

[2]. Bellido, A. N. (2004). Implementacion convertidor CC-CC con filtro de entrada y control robusto de la tension de salida. barcelona: ETSE.

[3]. Guapacha,J.B. Metodología para el aprendizaje de convertidores AC-DC, universidad tecnológica de Pereira, febrero 2013

[4]. Espinosa, H. M. (2009). Estudio y caraterizacion del par interruptor-diodo en un convertidor DC-DC. Cuernavacas,morales, mexico: Cenidex.

[5]. D. W, Hart, electrónica de potencia, , vol. 2, Madrid,2nd ed,2001,p.472

[6]. Herrera, S. C, Hurtado,S. O. (2015). Construccion de módulo convertido CC-CC elevador, para prácticas en el laboratorio del prgrama de ingenieria mecatronica. ,universidad tecnologica, pereiracolombia.

[7]. M. H, Rashid, Electrónica de potencia, circuito, dispositivos y aplicaciones, vol. 1, mexico,2nd ed,1995,p.721 